\title{
Bronchial asthma causing symptoms suggestive of angina pectoris
}

\author{
D. Kiss*, W. Veegh*, D. Schragel*, C. Bachl*, C. Stöllberger\#, K. Sert"*
}

Bronchial asthma causing symptoms suggestive of angina pectoris. D. Kiss, W. Veegh, D. Schragel, C. Bachl, C. Stöllberger, K. Sertl. C) ERS Journals Ltd 2003.

ABSTRACT: Many patients undergoing pharmacological stress echocardiography for assessing symptoms suggestive of coronary artery disease are found to have a normal test. This study was performed to evaluate whether asthma symptoms simulate angina pectoris.

A total 41 consecutive patients who had a negative pharmacological stress echocardiography and had been evaluated for angina pectoris were studied. Patients with previously known coronary artery disease or obstructive lung disease were excluded. Lung function testing was performed and the authors proceeded either with the administration of a bronchodilator or with methacholine challenge testing. A questionnaire was applied to assess the symptoms dyspnoea, chest tightness and substernal burning at the end of the challenge test. In addition, patients were asked if they were experiencing those symptoms that made them seek medical attention. From the 41 patients tested, 26 patients showed a significant bronchial hyperreactivity (mean \pm SD provocative concentration causing a $20 \%$ fall in the forced expiratory volume in one second $5.93 \pm 4.50 \mathrm{mg} \cdot \mathrm{mL}^{-1}$ ). A total of 20 patients confirmed having those symptoms that led to cardiac evaluation.

It was concluded that symptoms suggestive of angina pectoris might represent bronchial asthma. This should be considered in the differential diagnosis of angina pectoris.

Eur Respir J 2003; 21: 473-477.
*Dept of Internal Medicine, Sozialmedizinisches Floridsdorf and " Second Medical Dept, Krankenanastalt Rudolfstiftung, Vienna, Austria.

Correspondence: D. Kiss

SMZ Floridsdorf, Dept of Internal

Medicine

Hinaysgasse 1

1210 Vienna

Austria

Fax: 431275222109

E-mail: dora.kiss@flo.magwien.gv.at

Keywords: Angina pectoris asthma bronchial hyperreactivity echocardiography

stress

Received: December 132001

Accepted after revision: October 28 2002
Angina pectoris (literally, "strangling" in the chest) is a syndrome of recurrent discomfort in the chest or related areas associated with myocardial ischaemia or dysfunction but without myocardial necrosis [1]. Typical terms patients use to describe this discomfort are pressure, tightness in the chest, chest pain or a burning sensation. Symptoms may also be triggered by cold air or occur nocturnally. In some patients, myocardial ischaemia produces a significant transient decrease in the diastolic or systolic function of the ventricle that is responsible for exertional dyspnoea. In this case, dyspnoea is considered an angina equivalent $[2,3]$.

Patients presenting with symptoms suggestive of angina pectoris are usually referred for cardiac evaluation. The most widely used test to obtain objective evidence of myocardial ischaemia is treadmill exercise testing with a sensitivity of $60-70 \%$ and a specificity of $\sim 80 \%$ [4].

Patients with an inconclusive test result and patients who are incapable of performing a satisfactory treadmill exercise test have to be further assessed by specific noninvasive diagnostic tests, such as Thallium-201 perfusion scintigraphy, pharmacological stress echocardiography or single photon emission computed tomography to select those patients with a high probability of disease, where coronary angiography is reasonable and cost-effective [5]. Patients referred for these noninvasive tests have a low to intermediate pretest likelihood of coronary artery disease and therefore a considerable number of patients have negative test results [6]. This implies that some other condition is mimicking angina pectoris in these patients and there is a variety of differential diagnoses of angina pectoris in the textbooks. Bronchial asthma is not mentioned [1-3].

However, the sensation of chest tightness, chest pain and dyspnoea are also symptoms reported by asthmatics [7]. The triggers exertion, stress or exposure to cold are also associated with asthma attacks and alleviation by rest is described for exercise-induced asthma [8,9].

This study was designed to evaluate whether asthma is a differential diagnosis of angina pectoris.

\section{Methods}

\section{Subjects}

A total 41 consecutive patients (seven male, 34 female), aged 30-81 yrs, who had undergone pharmacological stress echocardiography for evaluation of symptoms suggestive of angina pectoris and had a normal test were examined.

The patients had been referred to the authors' outpatient clinic by their cardiologist, their internist, by the cardiology departments of two hospitals of the city of Vienna, Austria, or by the authors' Dept of Internal Medicine. Criteria for inclusion in this study were: recurrent angina-like chest symptoms, normal resting echocardiography and negative stress echocardiography within 3 weeks before their pulmonological evaluation. Patients were excluded if they had a previously diagnosed coronary heart disease or known obstructive lung disease.

After having obtained written consent to perform a pulmonary function study and eventually bronchial challenge testing, patients were interviewed about the onset and history of their symptoms, cardiac risk factors, smoking habits, asthma symptoms, atopy status, current medication and concomitant diseases. See table 1 for patient characterisation. 
Table 1.-Historical and clinical characteristics of the study patients

\begin{tabular}{lc} 
& Patients \\
\hline Patients total n & 41 \\
Age yrs mean \pm SD & $56 \pm 11.0$ \\
Male sex & 7 \\
Risk factors & 12 \\
Hypertension & 14 \\
Active smoker & 12 \\
Hyperlipidaemia & 5 \\
Family history of heart disease & 2 \\
Diabetes & \\
Symptoms & $16.5 \pm 15.6$ \\
Duration of symptoms months mean \pm SD & 20 \\
Chest pain & 39 \\
Chest tightness & 11 \\
Burning sensation in the chest & 29 \\
Dyspnoea during attacks & 23 \\
Exertional dyspnoea & 0 \\
Wheezing & 13 \\
Nocturnal symptoms & 6 \\
Cough & \\
\hline
\end{tabular}

\section{Stress echocardiography}

Pharmacological stress echocardiography was performed with the Sonos 5500 (Hewlett Packard, Andover, USA), which has an incorporated stress echocardiography module. All patients underwent pharmacological echocardiography with either dobutamine or dipyridamole, according to recommended standard protocols [10-12]. The patients were studied using an incremental regimen of $5,10,20,30,40 \mu \mathrm{g} \cdot \mathrm{kg}$ body weight ${ }^{-1} \cdot \mathrm{min}^{-1}$ of dobutamine every $3 \mathrm{~min}$ (12 patients) or a two-step dipyridamole protocol with a dosage $\leqslant 0.84 \mathrm{mg} \cdot \mathrm{kg}^{-1}$ (29 patients). Two-dimensional echocardiography and 12-lead electrocardiography was monitored during the infusion of the drug. If the predicted maximal cardiac frequency was not reached by the last increase in dosage, atropine $(\leqslant 1 \mathrm{mg})$ was administered. The target cardiac frequency was predicted by substracting age from 200 beats $\cdot \mathrm{min}^{-1}$ and was achieved by all patients who underwent the dobutamine protocol. Twodimensional images were analysed with reference to a 16segment model [13].

The stress echocardiogram was considered abnormal only if the applied drug induced a new wall motion abnormality as determined by review of the digitised echocardiographical images in a squad screen format and on videotape.

The presence or absence of pulmonary wheezing or other symptoms during the pharmacological stress test was documented.

Patients whose resting and stress echocardiography were classified normal and who passed the described inclusion and exclusion criteria were given an appointment at the authors' pulmonology laboratory.

\section{Lung function and challenge test}

The pulmonary function test was performed following American Thoracic Society (ATS) guidelines by a bodyplethysmograph (VMAX 2200; Sensormedics, Yorba Linda, CA, USA) measuring forced vital capacity (FVC), forced expiratory volume in one second (FEV1), total lung capacity (TLC) and residual volume (RV). FEV1 values $<80 \%$ in combination with FEV1/FVC values $<70 \%$ were considered to show bronchial obstruction [14]. In this case, patients were given an inhalative betamimetic agent (formoterol) and retested for reversibility after $15 \mathrm{~min}$. Reversibility was attested if the
FEV1 increased by $\geqslant 12 \%$ of the baseline value. Patients with a normal pulmonary function test continued their evaluation with bronchial challenge testing.

Challenge testing was performed by applying the tidal breathing method with doubling concentrations of methacholine up to a concentration of $20 \mathrm{mg} \cdot \mathrm{mL}^{-1}$, according to published guidelines [15]. Aerosol was inhaled by quiet tidal breathing through the mouth for $2 \mathrm{~min}$, using a noseclip. The FEV1 was measured before and 30 and $90 \mathrm{~s}$ after each inhalation. The challenge test was finished after a $20 \%$ fall of FEV1 compared to the baseline value or after completion of the concentration steps. A cut-off point of $16 \mathrm{mg} \cdot \mathrm{mL}^{-1}$ was used for the provocative concentration producing a $20 \%$ fall in the FEV1 (PC20) to discriminate hyperreactive from nonhyperreactive subjects $[15,16]$.

Immediately after ending the test, patients with a positive reaction were asked to answer the following questions concerning their symptoms. 1) Do you have trouble breathing? 2) Are you feeling tightness in your chest? 3) Are you feeling a burning sensation in your chest? 4) Are you feeling any symptoms at this moment, which have made you visit a doctor?

All patients were given an inhalative bronchodilator and hyperreactive patients were asked to perform spirometry measurements in 15-min intervals until they reached approximately their baseline FEV1 value.

The PC20 was determined by linear interpolation from the $\log 10$ concentration/response curve. Patients with a PC20 of $16 \mathrm{mg} \cdot \mathrm{mL}^{-1}$ or lower were classified as bronchial hyperreactive and were started on an inhalative asthma medication consisting of a combination of salmeterol and fluticasone twice daily and salbutamol p.r.n. Patients who showed a high level of hyperreactivity and who reported having frequent and severe attacks were additionally prescribed a course of oral steroids for 10-14 days (methylprednisolone $40 \mathrm{mg} \cdot \mathrm{day}^{-1}$ ). After starting asthma therapy, patients were rescheduled for a follow-up visit after 14-28 days. At this follow-up visit the patients were asked to answer following questions. 1) Concerning your prior complaints, are you feeling the same, better or worse than before having started the therapy? 2) Did the frequency of your symptoms change? If the answer is yes: is it more or less frequent? 3) Have your symptoms completely resolved?

Patients who did not perform bronchial challenge testing because of an abnormal lung function test were also started on the described therapy and rescheduled for a follow-up visit.

\section{Results}

The symptoms during the pharmacological stress test are described in table 2. None of the patients developed pulmonary wheezing, one patient complained about dyspnoea, and

Table 2.-Symptoms during the pharmacological stress echocardiography

Patients

Patients total $n$ 41

Symptoms

Headache

Palpitations

Chest pain

Chest tightness

Nausea

Dizziness

Wheezing

Dyspnoea

Asymptomatic
10

4

2

9

2

4

0

1 
nine patients complained about chest tightness. There was no difference in symptoms in patients who underwent dipyridamole stress echocardiography.

During the 8 months over which this study was performed at the authors' departments, there was a total number of 142 patients referred to pharmacological stress testing, including 59 patients with known coronary heart disease and/or abnormal resting echocardiogram. Of the remaining 83 patients, 12 showed a positive test result in terms of an ischaemic reaction and three had an inconclusive test. The remaining 68 patients with a negative stress test result were considered for the study. After excluding patients with known obstructive pulmonary disease and patients who did not suffer from recurrent symptoms, 41 patients qualified for inclusion in the study.

Of the 41 patients tested, 37 had a normal lung function test and were therefore eligible for bronchial challenge testing. From the remaining four patients, three had an FEV1/FVC of $<70 \%$, indicating bronchial obstruction, and one had hyperinflated lung volumes (103\% predicted TLC, 146\% pred RV). After bronchodilation, these patients showed no reversibility, making the diagnosis of so far undiagnosed chronic obstructive lung disease (COPD) likely.

Among the 37 patients who underwent challenge testing, 26 patients (mean age \pm sD $57.7 \pm 11.4$ yrs; five male, 21 female) responded to methacholine in terms of a decrease in FEV1 of $\geqslant 20 \%$ compared to the baseline value (mean PC20 5.93 $4.50 \mathrm{mg} \cdot \mathrm{mL}^{-1}$ ). Their lung function data, PC20 and symptoms are shown in table 3 .

From these 26 hyperreactive patients, 25 developed symptoms: 20 complained about dyspnoea, 25 developed the sensation of chest tightness and six reported feeling a burning sensation in their chest. A total 20 patients answered positively to the question that asked whether their symptoms made them seek medical attention. Among these patients, there were also two patients who had a history of allergic rhinitis, a condition that is often associated with bronchial hyperresponsiveness and predisposing for allergic asthma. One hyperreactive patient did not develop any symptoms and declined therapy. The other 25 hyperreactive patients and the four patients with the abnormal lung function tests were started on an anti-inflammatory and anti-obstructive therapy as described in the Methods section and rescheduled for a follow-up visit 2-4 weeks after their challenge test.

At their follow-up visit six of the 25 asthmatic patients reported a resolution and 18 reported a amelioration of their symptoms. The four patients with COPD reported no change.

\section{Discussion}

In the present study, patients with angina-like chest pain but normal pharmacological stress echocardiography for having their symptoms caused by bronchial asthma were examined. In their pulmonological evaluation performed by means of bronchial challenge testing, 26 of 41 patients were found to be hyperreactive, 25 were symptomatic during the test and 20 of them confirmed having those symptoms that made them seek medical attention. These results suggest that among patients with chest pain without evidence of coronary heart disease there are patients with bronchial asthma.

Of these 26 patients, two had a history of allergic rhinitis, which is often associated with bronchial hyperresponsiveness without any clinical evidence of bronchial asthma. Patients with allergic rhinitis are also predisposed to bronchial asthma and, considering their clinical presentation, bronchial hyperresponsiveness and their response to therapy, the authors found it justified to diagnose bronchial asthma in the two patients discussed.

Table 3. - Lung function data, provocative concentration of methacholine causing a $20 \%$ fall in the forced expiratory volume in one second ( $\left.\mathrm{FEV}_{1}, \mathrm{PC}_{20}\right)$ and symptoms during the challenge test and changes with asthma therapy in the 26 patients with bronchial hyperreactivity

\begin{tabular}{|c|c|c|c|c|c|c|}
\hline Patient no. & Sex & Age yrs & FEV1\% pred & $\mathrm{PC} 20 \mathrm{mg} \cdot \mathrm{mL}^{-1}$ & AP symptoms leading to evaluation & Better with therapy \\
\hline 1 & $\mathrm{~F}$ & 54 & 82 & 15.9 & Yes & Yes \\
\hline 5 & $\mathrm{~F}$ & 52 & 97 & 14.9 & Yes & Yes \\
\hline 7 & $\mathrm{~F}$ & 56 & 93 & 1.2 & Yes & Yes \\
\hline 9 & M & 32 & 88 & 7.4 & No & Declined therapy \\
\hline 10 & $\mathrm{~F}$ & 46 & 85 & 5.7 & Yes & Yes \\
\hline 12 & $\mathrm{~F}$ & 73 & 89 & 15.9 & Yes & Yes \\
\hline 13 & $\mathrm{~F}$ & 71 & 81 & 3.5 & Yes & Yes \\
\hline 14 & $\mathrm{~F}$ & 59 & 90 & 13.4 & Yes & Yes \\
\hline 15 & $\mathrm{~F}$ & 53 & 118 & 4.1 & No, but dyspnoea & Yes \\
\hline 16 & $\mathrm{~F}$ & 81 & 79 & 7.2 & Yes & Yes \\
\hline 18 & M & 53 & 94 & 6.7 & No, but dyspnoea & Yes \\
\hline 19 & $\mathrm{~F}$ & 73 & 122 & 1.6 & Yes & Yes \\
\hline 21 & $\mathrm{~F}$ & 60 & 119 & 6.6 & Yes & Yes \\
\hline 22 & $\mathrm{~F}$ & 66 & 116 & 10.0 & Yes & Yes \\
\hline 23 & $\mathrm{~F}$ & 45 & 95 & 4.3 & Yes & Yes \\
\hline 26 & M & 74 & 104 & 4.3 & Yes & Yes \\
\hline 27 & $\mathrm{~F}$ & 55 & 94 & 0.68 & Yes & Yes \\
\hline 29 & $\mathrm{~F}$ & 64 & 107 & 1.6 & Yes & Yes \\
\hline 31 & $\mathrm{~F}$ & 52 & 83 & 7.3 & Yes & Yes \\
\hline 33 & $\mathrm{~F}$ & 66 & 85 & 4.1 & Yes & Yes \\
\hline 34 & $\mathrm{~F}$ & 66 & 96 & 3.6 & Yes & Yes \\
\hline 36 & $\mathrm{~F}$ & 61 & 92 & 2.1 & No, but tightness and dyspnoea & Yes \\
\hline 38 & M & 39 & 112 & 3.6 & No, but tightness & Yes \\
\hline 39 & $\mathrm{~F}$ & 47 & 102 & 1.7 & Yes & No \\
\hline 40 & $\mathrm{~F}$ & 54 & 79 & 1.7 & Yes & Yes \\
\hline 41 & M & 49 & 114 & 5.1 & No, but tightness and wheezing & Yes \\
\hline
\end{tabular}

$\%$ pred: \% predicted; AP: angina pectoris; F: female; M: male. 
The evaluation of chest pain is a common clinical challenge. According to Bayes' theorem, females, whose coronary artherosclerotic disease prevalence in the general population of most Western countries is lower than that of males, tend to have more false-positive electrocardiographical exercise tests [3]. In addition, some other female attributes, either anatomical or physiological, appear to add to the number of falsepositive test results [17]. Dobutamine and dipyridamole stress echocardiography has been proven to be a reliable tool in detecting coronary artery disease in females. It is widely applied to stratify female patients with either abnormal or normal results of treadmill exercise testing and to avoid unnecessary cardiac catheterisation [18].

In a considerable number of patients, the majority of them being females, the clinical characteristics consistent with angina may be atypical or missing. This, and the abovementioned significantly lower positive predictive value of exercise treadmill testing in females, may explain the predominance of females in the patient group who had been referred to pharmacological stress echocardiography by their physicians.

Approximately $10 \%$ of patients referred for coronary angiography because of chest pain have angiographically normal coronary arteries and no other heart disease [4].

In further diagnostic evaluation, a variety of extracardial causes, such as gastro-oesophageal reflux disease, neuralgia, chest wall pain and vertebrogen pain have to be considered. After a thorough work-up excluding other diseases, there may still be patients whose symptoms remain unexplained. Patients with unexplained chest pain are significantly younger and more likely to be female. After their cardiac and, eventually, noncardiac evaluation they are often attributed psychiatric diagnoses, such as somatisation, anxiety disorders or depression $[19,20]$. Although they are assured by their physician that they could take up their normal physical activities, $\sim 50 \%$ of patients with unexplained chest pain are unable to work and describe their activity as being limited by chest pain [21].

When patients are asked to describe their symptoms, they have trouble discriminating chest pain from chest tightness or substernal burning or even dyspnoea. Different terms that are leading physicians in different clinical directions may be used synonymously by patients. The patients' vocabulary does not always meet the criteria for a typical clinical presentation.

The typical clinical features of asthma are recurrent episodes of dyspnoea, wheezing and cough, which may occur nocturnally or under physical or emotional stress and may be accompanied by nonrespiratory symptoms such as chest tightness or chest pain [7-9, 22, 23]. Some of the described symptoms, triggers and relievers are similar to those associated with angina pectoris and may therefore be misleading by suggesting a cardiac cause of the chest discomfort, especially to physicians who are cardiologically oriented. The patients referred to the authors' department for stress testing had been clinically evaluated by cardiologists, specialists for internal medicine and a cardiology department. This may explain why the investigative pathway chosen focused on heart disease. None of the patients had been evaluated by a pulmonologist.

The 41 patients in the present study presented with the predominant symptom of recurrent chest discomfort, 20 of them with chest pain which led to their cardiac evaluation. Twenty-nine patients reported having dyspnoea during their symptomatic episodes, only six patients complained about cough and none of the patients presented with or had a history of wheezing, which is generally considered a major symptom of asthma [3, 8, 9].

The patients' cardiac evaluation for ischaemic heart disease was normal. During the pharmacological stress echocardiography none of the patients developed pulmonary wheezing and only one patient of the nonhyperreactive group complained about dyspnoea.

Other possible causes for their symptoms, such as gastrooesophageal reflux disease, have not been investigated because this was not the objective of the present study. A total 26 patients were hyperreactive in the challenge test and, with regard to their clinical symptoms, 25 of them may be considered asthmatics. Only 11 patients had a normal pulmonary function test and negative challenge test. In epidemiological studies, the prevalence of bronchial hyperresponsiveness in the authors' geographical area was shown to be $\sim 12 \%$ and that of clinical asthma $5.2 \%[24,25]$.

The results of this study suggest that a significant number of unrecognised asthmatics are found among patients with angina-like chest pain but normal cardiac evaluation. It is concluded that bronchial asthma may present with chest pain or the isolated symptom of chest discomfort and should be included in the differential diagnosis of angina pectoris.

\section{References}

1. Ross J Jr. Assessment of cardial function and myocardial contractility. In: Schlant RC, Alexander RW, eds. Hurst's The Heart: Arteries and Veins. 8th Edn. New York, McGraw-Hill, 1994; p. 490.

2. Rutherford JD, Braunwald E. Chronic ischemic heart disease. In: Braunwald E, ed. Heart Disease. A Textbook of Cardiovascular Medicine. 4th Edn. Philadelphia, W.B. Saunders, 1992; p. 1293.

3. Selwyn AP, Braunwald E. Ischemic heart disease. In: Isselbacher KJ, Braunwald E, Wilson D, Martin JB, eds. Harrison's Principles of Internal Medicine. 13th Edn. New York, McGraw-Hill, 1994; p. 1079.

4. Ilsley C, Stockley A, Clitsakis D, Layton C. Normal coronary arteriogram. An avoidable test? Br Heart J 1982; 48: 580-583.

5. Hackshaw BT. Excluding heart disease in the patient with chest pain. Am J Med 1992; 92: 46S-51S.

6. Veegh W, Bachl C, Schragel D, Ernst G, Schneider B, Stöllberger C. The prognostic value of dobutamin stress echocardiography. Dtsch Med Wschr 1999; 124: 477-482.

7. Edmondstone WM. Chest pain and non-respiratory symptoms in acute asthma. Postgrad Med J 2000; 76: 413-414.

8. Weill H, Jones RN. Occupational pulmonary diseases. In: Fisherman AP, ed. Pulmonary Diseases and Disorders. New York, McGraw-Hill, 1988; p. 855.

9. Fraser RG, Paré JAP, Paré PD, Fraser SR, Genereux GP. Diagnosis Of Diseases Of The Chest. 3rd Edn. Philadelphia, W.B. Saunders Company, 1988.

10. Armstrong WF, Pellikka PA, Ryan T, Crouse L, Zoghbi W. Stress Echocardiography Task Force of the Nomenclature and Standards Committee of the American Society of Echocardiography. Stress Echocardiography: Recommendations for performance and interpretation of stress echocardiography. J Am Soc Echocardiogr 1998; 11: 97-104.

11. Cortigiani L, Picano E, Coletta C, et al. Safety, feasibility, and prognostic implications of pharmacologic stress echocardiography in 1482 patients evaluated in an ambulatory setting. Am Heart J 2001; 141: 621-629.

12. Pingitore A, Picano E, Varga A, et al. Prognostic value of pharmacological stress echocardiography in patients with known or suspected coronary heart disease. $\mathrm{J} \mathrm{Am}$ Coll Cardiol 1999; 34: 1769-1777.

13. Pingitore A, Picano E, Colosso MQ, et al. The atropine factor in pharmacologic stress echocardiography. Echo Persantine and Echo Dobutamine International Cooperative Study Groups. J Amer Coll Cardiol 1996; 27: 1164 1170 .

14. American Thoracic Society. Standardization of spirometry 1994 update. Am J Crit Care Med 1995; 152: 1107-1136. 
15. Sterk PJ, Fabbri LM, Quanjer PhH, et al. Airway Responsiveness. Standardized challenge testing with pharmacological, physical and sensitizing stimuli in adults. Eur Respir $J$ 1993; 6: Suppl. 16, 53-83.

16. Sunyer J, Munoz A and the Spanish group of the European asthma study. Concentrations of methacholine for bronchial responsiveness according to symptoms, smoking, and immunoglobulin $\mathrm{E}$ in a population-based study in Spain. $A m J$ Respir Crit Care Med 1996; 153: 1273-1279.

17. Barolsky SM, Gilbert CA, Faruqui A, Nutter DO, Schlant RC. Differences in electrocardiographic response to exercise of women and men: a non-Bayesian factor. Circulation 1979; 60: 1021-1027.

18. Ho YL, Wu CC, Huang PJ, et al. Assessment of coronary artery disease in women by dobutamine stress echocardiography: Comparison with Stress Thallium-201 SinglePhoton Emission Computed Tomography and Exercise Electrocardiography. Am Heart J 1998; 135: 655-662.

19. Flugelman MY, Weisstub E, Galun E, et al. Clinical, psychological and thallium stress studies in patients with chest pain and normal coronary arteries. Int J Cardiol 1991; 33: 401-408

20. Katon W, Hall ML, Russo J, Cormier L, Hollifield L,
Vitaliano PP. Chest pain: relationship of psychiatric illness to coronary arteriography results. Am J Med 1988; 84: 1-9.

21. Ockene IS, Shay MJ, Alpert JS, Weiner BH, Dalen JE. Unexplained chest pain with normal coronary arteriograms: a follow up study of functional status. N Engl J Med 1980; 303: $1249-1252$

22. Nudel DB, Diamaint S, Brady T, Jarenwattananon M, Buckley BJ, Gootman N. Chest pain, dyspnea on exertion, and exercise induced asthma in children and adolescents. Clin Pediatr (Phila) 1987; 26: 388-392.

23. Wiens L, Sabath R, Ewing L, Gowdamarajan R, Portnoy J, Scagliotti D. Chest pain in otherwise healthy children and adolescents is frequently caused by exercise-induced asthma. Pedatrics 1992; 90: 350-353.

24. Chinn S, Burney P, Jarvis D, Luczynska C, on behalf of the European Community Respiratory health Survey. Variation in bronchial responsiveness in the European Community Respiratory Health Survey (ECRHS). Eur Respir J 1997; 10: 2495-2501.

25. Popp W, Wagner C, Merkle M, Reiser K, Kiss D, Zwick H. Allergic rhinitis, respiratory obstruction and bronchial asthma in the Viennese population. Wien Klin Wochenschr 1993; 105: 377-381. 